\title{
Risk factors and biomarkers of life-threatening cancers
}

\section{Philippe Autier}

University of Strathclyde Institute of Global Public Health at iPRI, International Prevention Research Institute (iPRI, www.i-pri.org), Espace Européen d'Ecully, Bâtiment G, Allée Claude Debussy, Ecully ouest Lyon 69130, France

Correspondence to: Philippe Autier. Email: philippe.autier@i-pri.org

\begin{abstract}
There is growing evidence that risk factors for cancer occurrence and for cancer death are not necessarily the same. Knowledge of cancer aggressiveness risk factors (CARF) may help in identifying subjects at high risk of developing a potentially deadly cancer (and not just any cancer). The availability of CARFs may have positive consequences for health policies, medical practice, and the search for biomarkers. For instance, cancer chemoprevention and cancer screening of subjects with CARFs would probably be more ethical and cost-effective than recommending chemoprevention and screening to entire segments of the population. Also, the harmful consequences of chemoprevention and of screening would be reduced while effectiveness would be optimised. We present examples of CARF already in use (e.g. mutations of the breast cancer (BRCA) gene), of promising avenues for the discovery of biomarkers thanks to the investigation of CARFs (e.g. breast radiological density and systemic inflammation), and of biomarkers commonly used that are not real CARFs (e.g. certain mammography images, prostate-specific antigen (PSA) concentration, nevus number).
\end{abstract}

Keywords: biomarker, cancer, chemoprevention, phenotype, risk factor, screening

Published: $24 / 11 / 2015$

Received: 14/05/2015

ecancer 2015, 9:596 DOI: 10.3332/ecancer.2015.596

Copyright: $\odot$ the authors; licensee ecancermedicalscience. This is an Open Access article distributed under the terms of the Creative Commons Attribution License (http://creativecommons.org/licenses/by/3.0), which permits unrestricted use, distribution, and reproduction in any medium, provided the original work is properly cited. 


\section{Introduction}

The environmental, lifestyle, and genetic causes of cancer have received considerable attention over the past five decades. Hundreds of studies have unveiled some of the risks factors involved in the occurrence of cancer. By comparison, few studies have examined risk factors for being diagnosed with a life-threatening cancer versus being diagnosed with an indolent cancer that would never be life-threatening. In other words, what are the characteristics of subjects likely to be diagnosed with a 'pussy cat' cancer (i.e. non-life-threatening or easily curable) or with a 'tiger' cancer (i.e. deadly in the absence of an efficient treatment)? Do subjects diagnosed with a metastatic cancer present characteristics that distinguish them from subjects without cancer or from subjects with early-stage cancer, a part from attending screening? These characteristics may be linked to a variety of personal attributes, hereditary traits, lifestyle factors and exposures to substances or circumstances associated with a greater likelihood developing of a 'tiger' rather than of a 'pussy cat' cancer.

Cancers may be life-threatening either because they are diagnosed at a late stage, when metastases have spread into the lymph nodes or in distant organs, or because the cancer has an aggressive phenotype. Indeed, it is well documented that the more aggressive a cancer is, the greater the likelihood it will be diagnosed when at a late stage. However, a sizeable proportion of subjects dying from cancer are diagnosed with a cancer that is apparently still localised. Conversely, a fraction of subjects with advanced cancer survive for a long time even though they received the same amount of attention as other patients with the same stage of cancer.

Recent epidemiological and clinical studies have documented that risk factors for cancer occurrence and for cancer death are not necessarily the same, and that pre-diagnostic, non-tumour-related risk factors could be different for slow progressing or for aggressive cancers [1, 2]. Identification of subjects at higher risk to develop a potentially deadly cancer could thus be based on the recognition of specific personal genetic, lifestyle, or environmental characteristics, or on the measurement of non-tumour biomarkers, all factors that we term 'cancer aggressiveness risk factor' (CARF) hereafter.

\section{Risk factors for cancer occurrence and for cancer death}

Cancers affecting the same organ are no longer considered as a single disease entity. The wisdom that risk factors for cancer occurrence would be the same as for the risk for aggressive cancer or of cancer death is not correct. For instance, reproductive factors have a strong influence on the risk of breast cancer but little influence on the risk of breast cancer death [2]. Adiposity is associated with reduced breast cancer risk in premenopausal women. However, the risk of death from breast cancer in premenopausal women increases with adiposity [3]. High fertility is associated with reduced risk of breast cancer. However, women giving birth in their 40 s have become increasingly common, and breast cancer occurring in the first 2 years after childbirth is known to be more lethal [4]. Another example is smoking in prostate cancer. If smoking is not a risk factor for prostate cancer occurrence, it seems to be associated with the occurrence of fatal prostate cancer [5].

The search for hereditary, lifestyle, and environmental factors that would be involved in the occurrence of potentially life-threatening cancers has really started only after 2000 , mainly because of the longstanding false impression that risk factors for cancer occurrence and for cancer death were similar. In 1990, an International Agency for Research on Cancer (IARC, Lyon, France) Scientific Publication on the causes, occurrence, and control of cancer stated that 'the fact of death from cancer is rarely of interest in epidemiology' [6]. There was also the belief from the pre-screening era that any lesion that is labelled as 'cancer' by histological examination would necessarily be life-threatening.

\section{CARF, health policies, and patient management}

The availability of CARFs allowing risk stratification would have several implications for health policies and medical practice (Table 1).

Firstly, primary prevention efforts could concentrate on subjects with CARF(s). Personalised counselling based on the presence of CARF could be more effective than primary prevention campaigns spread over entire populations. 
Table 1. Examples of domains of application of cancer aggressiveness risk factors (CARF).

\begin{tabular}{|l|}
\hline Primary prevention \\
Definition of population subgroups most likely to benefit from primary prevention policies \\
Personalised prevention according to CARF presented by subjects \\
\hline Screening \\
Definition of population subgroups in which the balance between harms and benefits would be optimal \\
Personalised screening (age at start, method(s) of choice, intensity) according to CARFs presented by subjects \\
Identification of subjects with in benign lesion (e.g. breast hyperplasia) to develop a life-threatening invasive cancer \\
Identification of subjects with in situ or borderline cancers most likely to develop a life-threatening invasive cancer \\
Characterisation of subjects participating to screening most likely to develop and interval cancer \\
\hline Patient management \\
Selection of patients with in situ, borderline and early-stage cancer for active surveillance (no CARF) rather than \\
immediate treatment (CARF is present) \\
More intense follow-up of patients with apparently good prognosis cancer if early detection of relapse increases survival \\
Selection of subjects with apparently good prognosis cancer for the evaluation of adjuvant therapies \\
\hline Basic research \\
Knowledge of biological mechanisms involved in CARFs may lead to the discovery of: \\
biomarkers with better sensitivity and specificity for indentifying subjects at higher rish of potentially deadly cancer \\
new prevention, screening, and treatment modalities \\
\hline
\end{tabular}

Secondly, because the principal goal of cancer screening is the prevention of cancer death through the detection of cancers at an early, curable stage, CARF could allow the prioritisation of screening efforts towards subjects with CARF and avoid screening in subjects having a low probability to develop an aggressive cancer. CARF could boost the cost-effectiveness of screening because it could be directed to subjects at higher risk of cancer death and not just to subjects at higher risk of cancer. Moreover, if an efficient screening method exists, knowledge by subjects that they harbour a CARF might motivate them to participate in screening.

Third, subjects diagnosed with an apparently good prognosis cancer but who harbour a CARF could be at a higher risk of relapse, which could lead to recommending more intense management of these subjects. Using this logic, availability of CARF may help in selecting subjects with early stage cancer for inclusion in randomised trials for testing the efficacy of adjuvant therapy. CARF may provide useful information for the selection of patients with in situ, borderline or early stage cancer for active surveillance (e.g. if no CARF is present) or for immediate treatment (e.g. if CARF is present). For instance, a history of pregnancy in the two years preceding a diagnosis of breast cancer or being diabetic when breast cancer is found are two CARFs associated with poorer prognosis, even when the tumour has been detected at an early stage (e.g. size less than $20 \mathrm{~mm}$ and oestrogenic receptor positive) [4, 7, 8].

Fourth, the discovery of CARF can have an invaluable role in research on biological mechanisms involved in the occurrence of deadly cancers.

\section{When would CARF be most relevant?}

The availability of CARF may help to identify subjects with high or low probability of being diagnosed with an aggressive cancer.

Availability of CARF would be mostly valuable when the incidence of a cancer is much greater (say more than two times greater) than the mortality due to that cancer, meaning that the majority of cancers would not be a cause of death. Over the last 30 years, screening and the availability of imaging and biopsy methods allowing the detection of steadily smaller tumours has led to considerable increases in the incidence to mortality ratio because of over diagnosis. Over diagnosis is the detection of a lesion that has all the features of a cancer under the microscope but does not progress and will thus never be life-threatening. These cancers that do not clinically behave like cancers are pseudocancers whose frequency has been boosted by the advent of screening. Over diagnosis is common in breast, prostate, and thyroid cancer, and in cutaneous melanoma. Over diagnosis is known to be an issue for computerised tomography (CT) scan lung cancer 
screening. Over diagnosis also encompasses the detection of in situ or of borderline cancers, most of which will never transform into an invasive cancer or be life-threatening. For instance, before the mammography screening era, in situ breast cancers represented less than $5 \%$ of all breast cancers. In areas where mammography screening is widespread, 15 to $20 \%$ of breast cancers are in situ. Although we still have poor knowledge of the natural evolution of untreated in situ breast cancer [9], these lesions are nearly always treated. Treatment may be aggressive, with mastectomy (sometimes bilateral), radiotherapy, adjuvant chemotherapy and search for lymph node metastases despite that the lack of evidence on the most adequate management of these tumours. Because the finding of in situ breast cancer generally entails treatment, the mammography detection of these lesions is usually considered as over diagnosis [10].

Using this logic, focusing screening efforts on subjects with CARF is likely to increase the cost-effectiveness of screening and lower the harms due to screening. CARF may also assist in the prioritisation of referral to diagnosis and specialised care.

\section{The quest for CARF}

Generally speaking, studies done so far have not identified many CARFs that could be proposed for the risk stratification of subjects. However, several CARFs are already well documented and some promising avenues for research have been identified. We outline some examples here later.

\section{Hereditary factors}

Subjects with germline mutation associated with increased risk of cancer may also be at increased risk of aggressive cancer. The best known example are mutations of the BRCA gene that confers a higher lifetime risk of breast cancer, and breast cancers found in BRCA women tend to be more aggressive, with greater frequency of the triple negative phenotype [11].

\section{Screen detectability of cancer}

In the 1980s and 1990s, it was thought that screen-detected cancers would display signatures of their aggressiveness. For instance, images suggesting large cancers on mammograms or high serum concentration of biomarkers (e.g. the PSA) would reflect the presence of more aggressive cancer. However, if screen-detected cancer can indeed be fatal, these cancers are on average less aggressive and have a better prognosis that is independent of risk factors known to predict cancer survival. Conversely, cancers that were missed by screening or developed in the interval between two screening rounds (i.e. the interval cancers) are more aggressive and have a worse prognosis than screen-detected cancers. Large suspicious mammography images containing calcium deposits often indicate the presence of in situ cancer that is seldom life-threatening.

In the 1990s, small size, single institution studies suggested that the greater the increase in PSA level between two screening rounds, the more likely the presence of an aggressive prostate cancer (i.e. Gleason Score of 7 or more). It turned out that the reverse was true. For instance, a recent re-analysis of the prostate, lung, colorectal, and ovarian (PLCO) trial showed that the magnitude of changes in PSA level between screening rounds is not a predictor of cancer aggressiveness (Table 2).

Table 2. Risk of aggressive prostate cancer (Gleason score $\geq 7$ ) by PSA variation over time (after Boniol et al, 2015).

\begin{tabular}{|c|c|c|c|c|c|c|}
\hline \multirow{2}{*}{ PSA variation } & \multirow{2}{*}{ Subjects } & \multirow{2}{*}{ Prostate cancers } & \multirow{2}{*}{ Gleason score $\geq 7$} & \multirow{2}{*}{ OR $^{*}$} & \multicolumn{2}{|c|}{$\mathbf{9 5 \%}$ CI } \\
\cline { 4 - 7 } & & & & & Lower bound & Upper bound \\
\hline$<-50 \%$ & 872 & 25 & 16 & 1.75 & 0.33 & 1.71 \\
\hline$-50 \% ;-20 \%$ & 5199 & 263 & 149 & 1.00 & \multicolumn{2}{|c|}{ Reference } \\
\hline$-20 \% ;+20 \%$ & 15723 & 1424 & 806 & 0.98 & 0.82 & 1.19 \\
\hline$+20 \% ;+50 \%$ & 5697 & 659 & 371 & 0.95 & 0.75 & 1.19 \\
\hline$>+50 \%$ & 3766 & 381 & 214 & & \\
\hline
\end{tabular}

*Model adjusted for age and baseline PSA value. 
Hence, for two of the most common cancers, currently available screening methods are not optimal in their ability to early detect the most life-threatening cancers, and future research needs to test the capacity of new screening methods to specifically detect potentially deadly cancers when still at a curable stage.

\section{Radiological density of breasts}

The risk of breast cancer is four to five times greater in women with radiographically dense breasts than in women with little or no density in the breast [12,13]. Breast radiological density is an attractive CARF for several reasons. Cancer detection is less sensitive in radiographically dense breasts, and thus, interval cancers tend to be more common in women with dense breast [14-16]. Cancers found in dense breasts have a more aggressive phenotype and are more advanced than when found in fatty, non-dense breasts $[12,17,18]$. Hence, there is growing support for informing women with dense breasts about their higher risk of breast cancer and greater probability of false-negative mammographic examination. There is, however, no firm evidence that other breast examinations (e.g. ultrasonography) would improve the ability of screening to detect mammographically silent cancers that could be life-threatening. In any case, for legal and financial reasons, a growing number of states in the USA requires that radiologists notify women about the radiological density of their breasts (http://www.diagnosticimaging.com/ breast-imaging/breast-density-notification-laws-state-interactive-map).

\section{Obesity, diabetes, and low-grade systemic inflammation}

Cancers diagnosed among obese and diabetic subjects have a poorer prognosis, because they tend to be of more aggressive phenotype, are more advanced at diagnosis and are less sensitive to treatments [3, 19-25]. More advanced stages would not just be due to a greater difficulty to detect cancer in obese subjects or to lower propensity of these subjects who participate to screening. Mechanisms by which adiposity and glucose metabolism disorders would influence cancer phenotype are not well known. The low-grade inflammation that prevails in most obese and diabetic subjects could be the factor underlying the occurrence of more aggressive cancer.

Indirect evidence for a link between systemic inflammation and aggressive cancer phenotype is provided by prospective studies on vitamin $D$ concentration and the risk of subsequent cancer. Subjects with systemic inflammation have lower vitamin D levels [26], and low vitamin D levels are associated with more aggressive and more advanced breast, colorectal, and prostate cancer, and of cutaneous melanoma [27-31].

Studies like those on the toll-like receptor $4[25,32]$ represent significant strides in the understanding between inflammatory processes and cancer. It is hoped that the vast body of data accumulating on inflammation and cancer will end up in the discovery of biomarkers allowing the identification of subjects at high risk of aggressive cancer and in the discovery of new cancer treatment modalities based on the control of inflammatory processes.

\section{Nevus count and cutaneous melanoma}

The number and size of skin nevi is the strongest predictor of one's chance of being diagnosed with a melanoma. Skin self-surveillance and skin screening are usually recommended to subjects with numerous nevi. The incidence of melanoma has dramatically increased over the last four decades, in part because of greater exposure to ultraviolet radiation during holidays [33], and in part because of steadily increasing rates of nevus excision [34]. The expected reductions in the risk of melanoma death associated with the screening of subjects with numerous nevi rests on the caveat that these subjects would be at higher risk of melanoma death than subjects with few nevi. However, the few available data do not suggest a higher risk of melanoma death associated with increased number of nevi [35]. A high mitotic rate of melanoma cells is associated with a poorer prognosis. A recent study found no association between nevus count and the mitotic rate [36]. Hence, there is no evidence so far that a high nevus count could represent a CARF for melanoma and preferential screening of subjects with numerous nevi is probably no more effective than screening subjects with few nevi.

\section{Search for CARF in the context of cancer screening}

Subjects participating in screening are generally diagnosed with smaller and earlier-stage cancer than subjects not participating to screening. Cancer earliness may be due to the detection of cancers that would have been more advanced and more life-threatening if they had been symptomatic (i.e. lead time cancers). Cancer earliness may also be due to the detection of pseudocancers that would have never been 
symptomatic (i.e. length time cancers). Hence, a greater proportion of early-stage cancer in screened rather than in unscreened subjects may be linked to screening efficiency (ability to detect cancer at an early, asymptomatic stage) and to screening-induced over diagnosis. In addition, screen-detected cancers are less aggressive, while interval cancers are more aggressive. Finally, subjects not participating in screening are those who, in the absence of screening, would be at highest risk of being diagnosed with an advanced cancer and to die from it.

Because of the complex relationships between screening and cancer phenotype, the search for CARF must be aware of possible biases that could be introduced by the screening history of subjects.

\section{Ethics}

Targeted screening on the basis of CARF would probably be more ethical than recommending screening to entire segments of the population that are just defined by age and sex. The harmful consequences of screening would be reduced, while effectiveness would be optimised.

A key issue for the screening of breast, prostate, and lung cancer is that subjects whose lives are saved by screening are not necessarily the same subjects that incur the harmful consequences of screening. For instance, it was estimated for the United Kingdom that for one death prevented thanks to mammography screening, there are three women with over diagnosed cancer [10]. Because in the United Kingdom, mammography screening is proposed every three years, the ratio of three over diagnosed cancers to one life saved must be higher in most other countries where mammography screening is done every year or every two years. Hence, if a CARF allowed the risk stratification of women for their likelihood to have an indolent or an aggressive breast cancer, screening could be concentrated in women at high risk to have a potentially deadly breast cancer and probably abandoned in women at low risk of such cancer.

\section{Conclusion}

The possibility of identifying subjects at high or at low risk of developing a potentially deadly cancer may represent a new frontier in cancer research that would have many implications for screening policies, chemoprevention, and decision on treatment options for early-stage cancer which could integrate a better evaluation of the risk of relapse and the aspiration to avoid overtreatment.

\section{Conflicts of interest}

The authors declare that there is no conflicts of interest.

\section{Acknowledgments}

This work was presented at the WP2 EurocanPlatform meeting: European recommendations for biomarker-based chemoprevention trials, October 13-14, 2014, at the European Institute of Oncology, Milan, Italy.

\section{References}

1. Autier P (2012) Risk factors for breast cancer for women aged $\mathbf{4 0}$ to $\mathbf{4 9}$ years Ann Intern Med 157(7) 529 author reply DOI: 10.7326/0003-4819-157-7-201210020-00016 PMID: 23027325

2. Barnett GC, Shah $\mathrm{M}$ and Redman $\mathrm{K}$ et al (2008) Risk factors for the incidence of breast cancer: do they affect survival from the disease? J Clin Oncol 26(20) 3310-3316 DOI: 10.1200/JCO.2006.10.3168 PMID: 18612147 
3. Loi S, Milne RL and Friedlander ML et al (2005) Obesity and outcomes in premenopausal and postmenopausal breast cancer Cancer Epidemiol Biomarkers Prev 14(7) 1686-1691 DOI: 10.1158/1055-9965.EPI-05-0042 PMID: 16030102

4. Daling JR, Malone KE and Doody DR et al (2002) The relation of reproductive factors to mortality from breast cancer Cancer Epidemiol Biomarkers Prev 11(3) 235-241 PMID: 11895871

5. Giovannucci E, Liu Y and Platz EA et al (2007) Risk factors for prostate cancer incidence and progression in the health professionals follow-up study Int J Cancer 121(7) 1571-1578 DOI: 10.1002/ijc.22788 PMID: 17450530 PMCID: 2430098

6. Tomatis L, Aitio A and Day NE et al (1990) Cancer: Causes, Occurence and Control Lyon, France: World Health Organization International Agency for research on cancer

7. Kaplan MA, Pekkolay $Z$ and Kucukoner $M$ et al (2012) Type 2 diabetes mellitus and prognosis in early stage breast cancer women Med Oncol 29(3) 1576-1580 DOI: 10.1007/s12032-011-0109-4

8. Callihan EB, Gao D and Jindal S et al (2013) Postpartum diagnosis demonstrates a high risk for metastasis and merits an expanded definition of pregnancy-associated breast cancer Breast Cancer Res Treat 138(2) 549-559 DOI: 10.1007/s10549-0132437-x PMID: 23430224 PMCID: $\underline{3608871}$

9. Ward EM, DeSantis CE and Lin CC et al (2015) Cancer statistics: breast cancer in situ CA Cancer J Clin DOI: 10.3322/caac.21321 PMID: 26431342

10. Independent UK panel on breast cancer screening (2012) The benefits and harms of breast cancer screening: an independent review Lancet 380(9855) 1778-1786 DOI: 10.1016/S0140-6736(12)61611-0 PMID: 23117178

11. Boyle P (2012) Triple-negative breast cancer: epidemiological considerations and recommendations Ann Oncol 23 Suppl 6 vi7-12 DOI: $10.1093 / a n n o n c / m d s 187$ PMID: 23012306

12. Yaghjyan L, Colditz GA and Collins LC et al (2011) Mammographic breast density and subsequent risk of breast cancer in postmenopausal women according to tumor characteristics J Natl Cancer Inst 103(15) 1179-1189 DOI: 10.1093/jnci/djr225 PMID: 21795664 PMCID: $\underline{3149043}$

13. Boyd NF, Rommens JM and Vogt $\mathrm{K}$ et al (2005) Mammographic breast density as an intermediate phenotype for breast cancer Lancet Oncol 6(10) 798-808 DOI: 10.1016/S1470-2045(05)70390-9 PMID: 16198986

14. Mandelson MT, Oestreicher N and Porter PL et al (2000) Breast density as a predictor of mammographic detection: comparison of interval- and screen-detected cancers J Natl Cancer Inst 92(13) 1081-1087 DOI: 10.1093/inci/92.13.1081 PMID: 10880551

15. Pollan M, Ascunce $\mathrm{N}$ and Ederra $\mathrm{M}$ et al (2013) Mammographic density and risk of breast cancer according to tumor characteristics and mode of detection: a Spanish population-based case-control study Breast Cancer Res 15(1) R9 DOI: 10.1186/ bcr3380 PMID: 23360535 PMCID: $\underline{3672793}$

16. Porter GJ, Evans AJ and Cornford EJ et al (2007) Influence of mammographic parenchymal pattern in screening-detected and interval invasive breast cancers on pathologic features, mammographic features, and patient survival AJR Am J Roentgenol 188(3) 676-683 DOI: 10.2214/AJR.05.1950 PMID: 17312053

17. Aiello EJ, Buist DS and White E et al (2005) Association between mammographic breast density and breast cancer tumor characteristics Cancer Epidemiol Biomarkers Prev 14(3) 662-668 DOI: 10.1158/1055-9965.EPI-04-0327 PMID: 15767347

18. Sartor H, Zackrisson S and Elebro K et al (2015) Mammographic density in relation to tumor biomarkers, molecular subtypes, and mode of detection in breast cancer Cancer Causes Control 26(6) 931-939 DOI: 10.1007/s10552-015-0576-6 PMID: 25860114

19. Coughlin SS, Calle EE and Teras LR et al (2004) Diabetes mellitus as a predictor of cancer mortality in a large cohort of US adults Am J Epidemiol 159(12) 1160-1167 DOI: 10.1093/aje/kwh161 PMID: 15191933 
20. Berclaz G (2004) Body mass index as a prognostic feature in operable breast cancer: the International breast cancer study group experience Ann Oncol 15(6) 875-884 DOI: 10.1093/annonc/mdh222 PMID: 15151943

21. Yang XR, Chang-Claude $J$ and Goode EL et al (2011) Associations of breast cancer risk factors with tumor subtypes: a pooled analysis from the breast cancer association consortium studies J Natl Cancer Inst 103(3) 250-263 DOI: 10.1093/inci/dja526 PMCID: $\underline{3107570}$

22. Liao S, Li J, Wang L and Zhang Y et al (2010) Type 2 diabetes mellitus and characteristics of breast cancer in China Asian Pac J Cancer Prev 11(4) 933-937 PMID: 21133604

23. Phipps Al, Chlebowski RT and Prentice R et al (2011) Body size, physical activity, and risk of triple-negative and estrogen receptor-positive breast cancer Cancer Epidemiol Biomarkers Prev 20(3) 454-463 DOI: 10.1158/1055-9965.EPI-10-0974 PMID: $\underline{21364029}$ PMCID: $\underline{3064558}$

24. Li Z, Luo Y and Gong Y et al (2011) Clinical features and molecular phenotypes of breast cancer in patients with type-2 diabetes mellitus Asian Pac J Cancer Prev 12(9) 2183-2188

25. Yang $\mathrm{H}$, Wang $\mathrm{B}$ and Wang $\mathrm{T}$ et al (2014) Toll-like receptor 4 prompts human breast cancer cells invasiveness via lipopolysaccharide stimulation and is overexpressed in patients with lymph node metastasis PLoS One 9(10) e109980 DOI: 10.1371 / journal.pone.0109980 PMID: 25299052 PMCID: 4192367

26. Autier $\mathrm{P}$, Boniol $\mathrm{M}$ and Pizot $\mathrm{C}$ et al (2014) Vitamin D status and ill health: a systematic review Lancet Diabetes Endocrinol 2(1) 76-89 DOI: 10.1016/S2213-8587(13)70165-7 PMID: 24622671

27. Ng SW, Zaghloul S and Ali H et al (2011) Nutrition transition in the United Arab Emirates Eur J Clin Nutr 65(12) 1328-1337 DOI: 10.1038/ejcn.2011.135 PMID: 21772317 PMCID: $\underline{3304306}$

28. Yao S, Sucheston LE and Millen AE et al Pretreatment serum concentrations of 25-hydroxyvitamin D and breast cancer prognostic characteristics: a case-control and a case-series study PLoS One 6(2) e17251 PMID: 21386992 PMCID: $\underline{3046139}$

29. Peppone LJ, Rickles AS and Janelsins MC et al (2012) The association between breast cancer prognostic indicators and serum 25-OH vitamin D levels Ann Surg Oncol 19(8) 2590-2599 DOI: 10.1245/s10434-012-2297-3 PMID: 22446898 PMCID: 4158737

30. Fang F, Kasperzyk JL and Shui I et al (2011) Prediagnostic plasma vitamin D metabolites and mortality among patients with prostate cancer PLoS One 6(4) e18625 DOI: 10.1371/journal.pone.0018625 PMID: 21494639 PMCID: 3071841

31. Newton-Bishop JA, Beswick S and Randerson-Moor J et al (2009) Serum 25-hydroxyvitamin D3 levels are associated with breslow thickness at presentation and survival from melanoma J Clin Oncol 27(32) 5439-5444 DOI: 10.1200/JC0.2009.22.1135 PMID: 19770375 PMCID: $\underline{2773226}$

32. Bhatelia K, Singh K and Singh R (2014) TLRs: linking inflammation and breast cancer Cell Signal 26(11) 2350-2357 DOI: 10.1016/j. cellsig.2014.07.035 PMID: $\underline{25093807}$

33. IARC (1992) IARC Monographs on the Evaluation of Carcinogenic Risks to Humans Solar and Ultraviolet Radiation Lyon, France: International Agency for Research on Cancer 1017-1606 (Print)

34. Welch HG, Woloshin S and Schwartz LM (2005) Skin biopsy rates and incidence of melanoma: population based ecological study BMJ 331(7515) 481 DOI: 10.1136/bmj.38516.649537.E0 PMID: 16081427 PMCID: 1199022

35. Lin WM, Luo S and Muzikansky A et al (2015) Outcome of patients with de novo versus nevus-associated melanoma $\mathrm{J} A m$ Acad Dermatol 72(1) 54-58 DOI: 10.1016/ij.jaad.2014.09.028 PMID: 25440436

36. Shen S, Wolfe R and McLean CA et al (2014) Characteristics and associations of high-mitotic-rate melanoma JAMA Dermatol 150(10) 1048-1055 DOI: 10.1001/jamadermatol.2014.635 PMID: 25142970 